\title{
Fragilidade e fatores de risco associados em pacientes com doença renal crônica em hemodiálise
}

\author{
Frailty and associated risk factors in patients with chronic kidney \\ disease on dialysis
}

Gabriela Dutra Gesualdo (https://orcid.org/0000-0002-0125-6468) ${ }^{1}$ Juliana Gomes Duarte (https://orcid.org/0000-0001-7678-038X) ${ }^{2}$ Marisa Silvana Zazzetta (https://orcid.org/0000-0001-6544-767X) ${ }^{2}$ Luciana Kusumota (https://orcid.org/0000-0001-9290-3722) ${ }^{3}$ Fabiana de Souza Orlandi (https://orcid.org/0000-0002-5714-6890) ${ }^{2}$
${ }^{1}$ Escola de Enfermagem de Ribeirão Preto, Universidade de São Paulo (USP). Rodovia Washington Luís, km 235, SP-310. 13565-905 São Carlos SP Brasil.

fabi_ferreira@yahoo.com.br ${ }^{2}$ Departamento de

Gerontologia, Universidade Federal de São Carlos. São Carlos SP Brasil.

${ }^{3}$ Departamento de Enfermagem Geral e Especializada, USP. São Paulo SP Brasil.

\begin{abstract}
The objective of this article is to determine the relationship between frailty and socio-demographic/clinical characteristics in patients with chronic kidney disease on dialysis. A cross-sectional study was conducted with 107 participants. Descriptive, correlation and logistic regression analyses were performed, with the level of significance set to $5 \%(p<0.05)$. The prevalence of frailty was $47.66 \%$. Frailty was negatively correlated with cognition $(r=-0.30 ; p=0.002)$, functioning on instrumental activities of daily living $(r=-0.41 ; p=0.000)$ and hematocrit level $(r=-0.19 ; p=0.04)$. The proportion of frailty increased with the age of the participants $(O R=$ 1.03; 95\%CI: 1.004-1.069; $p=0.02$ ). Individuals with chronic kidney disease on dialysis had high percentages of frailty, which was associated with an older age and correlated with cognition, functioning on instrumental activities of daily living and a lower hemotocrit level.
\end{abstract}

Key words Adult Health, Health of the Elderly, Frailty, Chronic Kidney Failure, Dialysis
Resumo O objetivo deste artigo é verificar a relação entre fragilidade e os aspectos sociodemográficos e clínicos de pacientes com doença renal crônica em hemodiálise. Estudo transversal, conduzido com 107 participantes. Realizou-se análise descritiva, correlação e regressão logística. Os resultados foram considerados significativos com p-valor $\leq$ 0,05. A prevalência de fragilidade correspondeu a 47,66\%, correlacionou-se negativamente com a cognição $(r=-0,30$; $p$-valor $=0,002)$, funcionalidade para atividades instrumentais de vida diária $(r=-0,41 ; p$-valor $=0,000)$ e com o nível de hematócrito $(r=-0,19$; $p$-valor $=0,04)$. Constatou-se a maior proporção de fragilidade em participantes com maior idade (OR = 1,03; IC95\% 1,004-1,069; $p=0,02$ ). Os participantes com doença renal crônica em hemodiálise apresentaram elevados percentuais de fragilidade, associada a maior idade e correlacionada a cognição, funcionalidade para atividades básicas de vida diária e menor nivel de hematócrito.

Palavras-chave Saúde do Adulto, Saúde do Idoso, Idoso Fragilizado, Insuficiência Renal Crônica, Diálise Renal 


\section{Introdução}

As doenças crônicas (DC) requerem intervenções, associadas à mudança de estilo de vida, em um processo de cuidado contínuo que nem sempre leva à cura. Estas constituem um problema de saúde de grande magnitude, correspondendo a $72 \%$ das causas de morte ${ }^{1}$. Neste contexto, a Doença Renal Crônica (DRC) surge como um sério problema de saúde nas populações, e pode ser considerada uma "epidemia" em crescimento ${ }^{2}$.

A DRC é definida como uma anormalidade da estrutura e/ou função renal, presente por mais de três meses, com implicações para a saúde. É preciso esclarecer a causa da doença, bem como definir seu estágio, com isso é possível identificar os riscos de desfechos adversos, relacionados ao comprometimento renal e ao óbito ${ }^{3}$.

O paciente com DRC em hemodiálise passa por graves mudanças na vida social, no trabalho, nos hábitos alimentares e na vida sexual, que acarretam alterações na sua integridade física e emocional. Essa condição ocasiona prejuízo corporal e limitações, pois, em geral, há afastamento do paciente de seu grupo social, de seu lazer e, às vezes, da própria família. Esses fatores podem tornar o paciente frágil e conduzir a um agravamento da sua qualidade de vida ${ }^{4}$.

A síndrome de fragilidade é de natureza clínica e multidimensional, caracterizada pelo aumento da vulnerabilidade a estressores, com diminuição da força, resistência e função fisiológica; com consequente risco de eventos adversos para a saúde, como dependência, hospitalização, institucionalização e mortalidade ${ }^{5,6}$.

Possuir doença renal aumenta muito as chances de fragilidade quando comparados aos indivíduos com função renal normal ou portadores de outras doenças crônicas (por exemplo, diabetes, doença pulmonar obstrutiva crônica, câncer e artrite reumatoide) $)^{7}$. Os pacientes com DRC apresentam redução da qualidade de vida e da cognição, diminuição da atividade física, perda da massa muscular e alta prevalência de sintomas como fadiga, náuseas e anorexia8.

A prevalência de fragilidade aumenta à medida que a Taxa de Filtração Glomerular (TFG) diminui, $14 \%$ dos pacientes com DRC são frágeis, em contrapartida, $10 \%$ dos idosos da comunidade apresentam o fenótipo de fragilidade ${ }^{9}$. Há uma alta prevalência entre os pacientes que iniciam diálise, sendo a fragilidade um construto importante nesta dimensão, pois está altamente associada aos desfechos adversos ${ }^{10}$.

A identificação da fragilidade e dos seus fatores de risco associados à pacientes com DRC em hemodiálise permite o aprimoramento da assistência da rede pública e privada visando intervenções mais concretas e viáveis de ser operacionalizadas. Ademais este estudo justificou-se na importância de se investigar, no Brasil, pois estratégias que visam o controle da síndrome de fragilidade podem possibilitar melhores condições de saúde.

Diante ao exposto, são relevantes estudos que identifiquem pacientes com DRC em condições de pré-fragilidade e fragilidade no contexto da hemodiálise, especialmente na população brasileira, assim como sua relação com os aspectos sociodemográficos e clínicos, considerando a elevada prevalência desta síndrome nesse cenário. Sendo assim, o objetivo deste estudo foi verificar a relação entre fragilidade e os aspectos sociodemográficos, clínicos de pacientes com doença renal crônica em hemodiálise.

\section{Método}

Trata-se de um estudo quantitativo, observacional e descritivo de corte transversal, conduzido com pacientes com DRC em uma Unidade de Terapia Renal Substitutiva do interior do Estado de São Paulo, região sudeste do Brasil.

A amostra foi não probabilística de conveniência, totalizando 107 participantes. A coleta ocorreu entre setembro e novembro de 2014. Foram considerados como critérios de inclusão: ter idade igual ou superior a 18 anos; ter diagnóstico médico de DRC e estar em hemodiálise há no mínimo seis meses. Foram excluídos da amostra os participantes que possuíam sequelas de Acidente Vascular Encefálico (AVE) e/ou com graves déficits de visão ou audição. Os dados foram coletados em uma sala privativa da própria unidade, na qual tiveram duração média de 1 hora, previamente a sessão de hemodiálise, foram aplicados o Instrumento de Caracterização do Participante, o Fenótipo de Fragilidade de Fried, o Exame Cognitivo de Addenbrooke-Revisado (ACE-R) e a Escala de Lawton.

Realizou-se inicialmente a avaliação das condições sociodemográfica e clínica dos participantes, utilizou-se um instrumento elaborado pelo grupo de pesquisa que foi construído para uso científico, a fim de pesquisa e utilizado em outros estudos, ele era composto por dados sociodemográficos e econômicos (sexo, idade, cor da pele referida, escolaridade e renda familiar), dados clínicos (tempo de hemodiálise e nível de hematócrito).

A cognição foi avaliada através do ACE- $R$ que é uma bateria para avaliação cognitiva que 
pode ser aplicada em adultos e idosos com elevada sensibilidade e especificidade para detectar demência em estágio leve e particularmente útil para diferenciar a doença de Alzheimer da demência frontotemporal. Contém cinco escores, cada um representando um domínio cognitivo. O escore geral do ACE-R vai de 0 a 100 pontos, podendo os domínios de orientação/atenção (18 pontos), memória (26 pontos), fluência verbal (14 pontos), linguagem (26 pontos) e habilidades visuais espaciais (16 pontos) serem avaliados individualmente. As notas de corte para a bateria completa e para cada domínio, incluindo o Mini Exame do Estado Mental (MEEM), inserido no ACE-R, foram definidas como: $<78$ pontos para a bateria completa, $<17$ pontos para orientação/ atenção, $<15$ pontos para memória, $<8$ pontos para fluência verbal, $<22$ pontos para linguagem, $<13$ pontos para habilidade visual construtiva e $<25$ pontos para o MEEM $^{11}$.

Para a avaliação das atividades instrumentais de vida diária foi utilizado a Escala de Lawton que avalia o desempenho funcional de adultos e idosos em termos de atividades instrumentais que possibilitam que o mesmo mantenha uma vida independente. As tarefas avaliadas são: usar o telefone, utilizar transporte, fazer compras, preparar refeições, arrumar a casa, fazer trabalhos manuais domésticos, manusear medicamentos e cuidar das finanças. Sua pontuação varia de 7 a 21 , sendo que pontuação igual a 7 caracteriza o indivíduo como dependente total, acima de 7 e menor que 21 , indicam dependência parcial e 21 pontos caracteriza-se como independente ${ }^{12}$.

Verificou-se a presença da síndrome de fragilidade através dos cinco itens descritos como componentes do fenótipo de fragilidade ${ }^{6}$ :

a) autorrelato de perda de peso corporal não intencional no último ano ( $>4,5 \mathrm{~kg}$ ou $>5 \%)$;

b) sensação de exaustão, avaliado por duas questões: "Com que frequência na última semana sentiu que tudo que fez exigiu um grande esforço?" e "Com que frequência na última semana, sentiu que não conseguiria levar suas coisas adiante?". Quando os participantes declararam "Na maioria das vezes", ou "Sempre", em pelo menos uma das questões pontuou-se como frágil;

c) baixa força de preensão palmar, avaliada pelo dinamômetro manual (Modelo Grip, fabricante SAEHAN ${ }^{\circledR}$ ), no membro superior onde não haja fístula arteriovenosa, solicitando ao avaliado que por três vezes exercesse a maior força possível, sendo indicativo de fragilidade participantes no primeiro quintil, após ajuste do resultado para sexo e índice de massa corporal; d) diminuição da velocidade da marcha, avaliada utilizando um cronômetro para medir o tempo gasto para percorrer um percurso de $4,6 \mathrm{~m}$. O resultado foi a média de três medidas consecutivas (em segundos), ajustadas de acordo com o sexo e a altura;

e) baixa prática de atividade física, avaliado por meio do autorrelato à pergunta “ $\mathrm{O}(\mathrm{a})$ senhor(a) acha que faz menos atividades físicas do que há doze meses atrás?", sendo considerado indicativo de fragilidade participantes que declararam "Sim".

A pontuação em três ou mais critérios significa a condição de fragilidade, um ou dois critérios o indivíduo é considerado pré-frágil, e quando não há pontuação em nenhum dos critérios ele é considerado não frágil ${ }^{6}$.

As análises realizadas foram descritiva com informações de tendência central (média, mediana, mínimo e máximo) e medidas de dispersão (desvio-padrão); coeficiente de correlação de Spearman entre a pontuação do Fenótipo de fragilidade e as variáveis sociodemográficas e clínicas, neste estudo a magnitude das correlações ${ }^{13}$ foi classificada em fraca $(<0,3)$; moderada $(0,3$ a $0,59)$, forte $(0,6$ a 0,9$)$ e perfeita $(1,0)$; e regressão logística para analisar os efeitos das variáveis sociodemográficas e clínicas independentes sobre a fragilidade.

O nível de significância utilizado para os testes estatísticos foi $\alpha=0,05$, ou seja, foram considerados significativos onde $\mathrm{p}$-valor $\leq 0,05$.

O projeto foi submetido ao Comitê de Ética em Pesquisa com Seres Humanos da Universidade Federal de São Carlos (UFSCar) e foi aprovado.

\section{Resultados}

Dentre os 107 entrevistados, a maioria era do sexo masculino $(67,30 \%)$, a escolaridade variou de 0 a 20 anos, sendo o tempo médio 7,26 anos. $O$ tempo médio de hemodiálise foi de 48,91 $(46,81)$ meses (Tabela 1).

A prevalência da condição de fragilidade correspondeu a $47,66 \%(n=51)$, enquanto a préfragilidade representou $44,85 \%(n=48)$ e não frágeis foram $7,49 \%(n=8)$.

Os participantes que apresentaram maior nível de fragilidade tiveram pior desempenho na avaliação cognitiva $(r=-0,30 ; p$-valor $=0,002)$, na funcionalidade para atividades instrumentais de vida diária $(\mathrm{r}=-0,41$; $\mathrm{p}$-valor $=0,000)$ e menor nível de hematócrito $(\mathrm{r}=-0,19 ; \mathrm{p}$-valor $=$ 0,04) (Tabela 2). 
Tabela 1. Distribuição dos pacientes com doença renal crônica, segundo características sociodemográficas e clínicas (n = 107). São Carlos, SP, 2018.

\begin{tabular}{|c|c|c|c|c|c|c|}
\hline Variável & Média (dp $\dagger)$ & Mediana & $\begin{array}{c}\text { Variação } \\
\text { observada }\end{array}$ & $\begin{array}{l}\text { Distribuição } \\
\text { em categorias }\end{array}$ & $\mathbf{n}$ & $\%$ \\
\hline \multirow[t]{2}{*}{ Sexo } & -- & -- & -- & Masculino & 72 & 67,30 \\
\hline & & & & Feminino & 35 & 32,70 \\
\hline \multirow[t]{7}{*}{ Idade (anos) } & $54,30(14,85)$ & 56,00 & $22-85$ & $20-29$ & 7 & 6,54 \\
\hline & & & & $30-39$ & 15 & 14,01 \\
\hline & & & & $40-49$ & 22 & 20,56 \\
\hline & & & & $50-59$ & 18 & 16,82 \\
\hline & & & & $60-69$ & 25 & 23,36 \\
\hline & & & & $70-79$ & 18 & 16,82 \\
\hline & & & & $80-89$ & 2 & 1,89 \\
\hline \multirow{6}{*}{$\begin{array}{l}\text { Escolaridade } \\
\text { (anos) }\end{array}$} & $7,26(4,10)$ & 8,00 & $0-20$ & 0 & 3 & 2,80 \\
\hline & & & & $1-4$ & 41 & 38,31 \\
\hline & & & & $5-8$ & 18 & 16,82 \\
\hline & & & & $9-12$ & 16 & 14,95 \\
\hline & & & & $13-16$ & 28 & 26,16 \\
\hline & & & & $17-20$ & 1 & 0,96 \\
\hline \multirow{6}{*}{$\begin{array}{l}\text { Tempo de } \\
\text { Hemodiálise (em } \\
\text { meses) }\end{array}$} & $48,91(46,81)$ & 36,00 & $6-264$ & $6 \vdash 18$ & 26 & 24,30 \\
\hline & & & & $18 \mathrm{~F} 30$ & 23 & 21,50 \\
\hline & & & & $30 \mathrm{~F} 42$ & 8 & 7,48 \\
\hline & & & & $42 \mathrm{~F} 54$ & 12 & 11,21 \\
\hline & & & & $54 \mathrm{~F} 66$ & 12 & 11,21 \\
\hline & & & & $66 \mathrm{H}^{264}$ & 26 & 24,30 \\
\hline
\end{tabular}

Tabela 2. Coeficiente de Correlação Spearman entre o fenótipo de fragilidade e as variáveis sociodemográficas e clínicas de pacientes com doença renal crônica. São Carlos, SP, 2018.

\begin{tabular}{lcccc}
\hline & Idade & Cognição (ACE-R) & $\begin{array}{c}\text { Funcionalidade } \\
\text { (Lawton) }\end{array}$ & Hematócrito \\
\hline Fenótipo & & & & $-0,41$ \\
$\mathrm{r} \dagger$ & $-0,15$ & $-0,30$ & 0,19 \\
$\mathrm{p} \ddagger$ & 0,11 & 0,002 & 0,000 & 107 \\
$\mathrm{n} \S$ & 107 & 107 & 107 &
\end{tabular}

Os pacientes mais velhos têm maior chance de serem frágeis. Cada ano de vida aumenta em $3 \%$ a chance de serem frágeis. As demais variáveis estudadas não apresentaram relação significante com o risco de fragilidade (Tabela 3 ).

Apenas as variáveis raça, renda per capita, tempo de hemodiálise, idade e cognição (ACE -R), se associaram ao instrumento de fragilidade, com $\mathrm{p}$-valor $\leq 0,20$ e entraram no modelo, executando-se as colineares. Por meio do método de seleção Backward, foram eliminadas as variáveis independentes que conjuntamente obtiveram $\mathrm{p}$ valor $>0,05$.

\section{Discussão}

A palavra fragilidade tem sido empregada para distinguir um grupo de pessoas mais fracas e mais vulneráveis. Estudos recentes nacionais e internacionais têm contribuído para a melhor definição das propriedades clínicas e fisiológicas da fragilidade, destacando a vulnerabilidade do idoso frágil e seus desfechos adversos ${ }^{7-14}$.

Embora os pacientes com DRC em hemodiálise em certas ocasiões apresentem clinicamente sinais e sintomas compatíveis com a fragilidade, ainda há escassez de informações sobre os fato- 
Tabela 3. Análise de regressão logística multivariada de possíveis fatores associados à fragilidade pelo fenótipo de fragilidade de pacientes com doença renal crônica. São Carlos, SP, 2018.

\begin{tabular}{llccc}
\hline \multicolumn{1}{c}{ Variáveis } & \multicolumn{1}{c}{ Categorias } & p-valor & O.R. $\dagger$ & IC95\% O.R. \\
\hline Raça & Branca (ref) & 0,44 & 0,71 & $0,30-1,69$ \\
& Não branca & & & \\
Renda per capita & Variável contínua & 0,56 & 1,08 & $0,82-1,40$ \\
Idade & Variável contínua & 0,02 & 1,03 & $1,004-1,069$ \\
Tempo de hemodiálise & Variável contínua & 0,07 & 0,99 & $0,98-1,00$ \\
Cognição (ACE-R) & Variável contínua & 0,67 & 0,99 & $0,98-1,01$ \\
\hline
\end{tabular}

†OR (OddsRatio) = Razão de chances para a fragilidade; ¥IC95\% OR = Intervalo de 95\% de confiança para a razão de risco.

res sociodemográficos e clínicos que podem estar associados $^{15}$.

A identificação da fragilidade e dos seus fatores preditores, no ambiente de cuidados de diálise tem o potencial de melhorar a identificação dos pacientes que estão em risco de desfechos adversos, e podem se beneficiar com intervenções destinadas a prevenir seu declínio ${ }^{8}$.

$\mathrm{Na}$ presente pesquisa identificamos uma grande parcela de pacientes com DRC em hemodiálise frágeis, e a síndrome de fragilidade foi relacionada com déficit cognitivo e funcional, menor nível de hematócrito e idade.

Diversos estudos nacionais e internacionais corroboraram com esta investigação, no que diz respeito a maior prevalência de fragilidade nessa população ${ }^{7,16-22}$. Os fatores de risco associados identificados por outros autores foram doença vascular periférica, doenças cardíacas, raça negra e menor concentração de albumina sérica ${ }^{15}$; mortalidade e hospitalização ${ }^{8}$; número de internações e risco de quedas ${ }^{17}$; diabetes mellitus $^{18}$; alta TFG $^{19}$; uso de vitamina $\mathrm{D}$ e paratormônio ${ }^{7}$; baixo desempenho físico e severidade da $\mathrm{DRC}^{20}$; pior qualidade de vida, sexo e idade ${ }^{21}$.

Porém, ressalta-se que a prevalência de fragilidade, obtida na presente investigação, foi superior a inquéritos com participantes da comunidade, aspecto que sugere risco à saúde, implicações para os serviços de saúde e sobrecarga aos profissionais atuantes em centros de diálise.

A limitação desta pesquisa diz respeito ao desenho transversal do estudo que impossibilita a identificação da precedência temporal dos fatores estudados. Sendo assim, a realização de investigações longitudinais neste cenário faz-se necessária, para maior aprofundamento e compreensão da gênese desta síndrome.

Apesar disso, acredita-se que a identificação das condições de saúde, relacionadas às condições de pré-fragilidade, pode favorecer o planejamento e implementação da assistência aos adultos e idosos neste contexto, tendo em vista as repercussões advindas desta síndrome.

Considerando a escassez de estudos nacionais, esta investigação pretende contribuir para ampliação do conhecimento dos fatores sociodemográficos e clínicos relacionados à fragilidade no contexto nefrológico.

Deste modo, estes dados podem subsidiar ações em saúde preventivas, direcionando o cuidado, a fim de postergar sua evolução. Neste contexto, salienta-se ainda a necessidade de reflexão do sistema de saúde sobre a rede de atenção ao adulto e idoso com DRC, para atender às necessidades de saúde, nos diversos níveis de atenção, considerando a maximização dos agravos à saúde advindas desta síndrome.

\section{Conclusão}

A prevalência do estado de fragilidade em pacientes com DRC em hemodiálise foi elevada, correlacionada negativamente com a cognição, funcionalidade pata atividades instrumentais de vida diária e nível de hematócrito, sendo associado à maior idade. 


\section{Colaboradores}

GD Gesualdo contribuiu substancialmente para o planejamento, análise e interpretação dos dados. Além disso, contribuiu significativamente na elaboração do artigo. Também participou da aprovação da versão final do manuscrito. JG Duarte contribui significativamente na elaboração do artigo e na revisão crítica do conteúdo. MS Zazzetta contribuiu substancialmente na elaboração do artigo e na revisão crítica do conteúdo. L Kusumota contribuiu substancialmente na elaboração do artigo e na revisão crítica do conteúdo. FS Orlandi contribuiu substancialmente para a concepção, planejamento, análise e interpretação dos dados. Além disso, contribuiu significativamente na elaboração do artigo e na revisão crítica do conteúdo. Todas as autoras participaram da aprovação da versão final do manuscrito.

\section{Agradecimentos}

À Coordenação de Aperfeiçoamento de Pessoal de Nível Superior (CAPES) pelo financiamento da pesquisa.

\section{Referências}

1. Brasil. Ministério da Saúde (MS). Diretrizes para o cuidado das pessoas com doenças crônicas nas redes de atenção à saúde e nas linhas de cuidado prioritárias. Brasília: MS; 2013.

2. Siveiro PCL, Machado PJ, Rodrigues PN. Doença renal crônica: um agravo de proporções crescentes na população brasileira. Belo Horizonte:CEDEPLAR/UFMG; 2013.

3. Kidney Disease: Improving Global Outcomes (KDIGO) CKD Work Group. KDIGO 2012 Clinical Practice Guideline for the Evaluation and Management of Chronic Kidney Disease. Kidney Int 2013; 3(Supl. 1) $1-163$.

4. Oliveira SG, Marques IR. Sentimentos do paciente portador de doença renal crônica sobre a autoimagem. Rev Enferm UNISA 2011; 12(1):38-42.

5. Morley JE, Vellas B, Van Kan GA, Anker SD, Bauer JM, Bernabei R, Cesari M, Chumlea WC, Doehner W, Evans J, Fried LP, Guralnik JM, Katz PR, Malmstrom TK, McCarter RJ, Robledo LMG, Rockwood K, Von Haehling S, Vandewoude MF, Walston J. Frailty consensus: a call to action. J Am Med Dir Assoc 2013; 14(6):392-397.

6. Fried LP, Ferrucci L, Darer J, Williamson JD, Anderson G. Untangling the concepts of disability, frailty, and comorbidity: implications for improved targenting and care. J Gerontol A Biol Sci Med Sci 2004; 59(3):255-263.

7. Mansur HN, Damasceno VO, Bastos MG. Prevalência da fragilidade entre os pacientes com doença renal crônica em tratamento conservador e em diálise. $J$ Bras Nefrol 2012; 34(2):153-160.

8. Johansen KL, Chertow GM, Jin C, Kutner NG. Significance of frailty among dialysis patients. J Am Soc Nephrol 2007; 18(11):2960-2967.

9. Johansen KL, Delgado C, Bao Y, Kurella Tamura M. Frailty and dialysis initiation. Semin Dial 2013; 26(6):690-696.

10. Walker SR, Gill K, Macdonald K, Komenda P, Rigatto C, Sood MM, Bohm CJ, Storsley LJ, Tangri N. Association of frailty and physical function in patients with non-dialysis CKD: a systematic review. BMC Nephrology 2013; 14:228.

11. Carvalho VA, Barbosa MT, Caramelli P. Brazilian version of Addenbrooke's Cognitive Examination in the diagnosis of mild Alzheimer Disease. Cog Behav Neurol 2010; 23(1):8-13.

12. Lawton MP, Brody E. Assessment of older people: self-maintaining and instrumental activities of daily living. The Gerontologist 1969; 9(3):179-186.

13. Levin J, Fox JA, Forde DR. Estatística para ciências humanas. 11ª ed. São Paulo: Pearson; 2012. 
14. Kim JC, Kalantar-Zadeh K, Kopple JD. Frailty and protein-energy wasting in elderly patients with end stage kidney disease. J Am Soc Nephrol 2013; 24(3):337-351.

15. Kutner NG, Zhang RMS, Huang Y, McClellan WM, Soltow QA, Lea J. Risk factors for frailty in a large prevalent cohort of hemodialysis patients. Am J Med Sci 2014; 348(4):277-282.

16. Willhelm-Lenn ER, Hall YN, Tamura MK, Chertow GM. Frailty and chronic kidney disease: the Third National Health and Nutrition Evaluation Survey. Am J Med 2009; 122(7):664-671.

17. McAdams-Demarco MA, Suresh S, Law A, Salter ML, Gimenez LF, Jaar BG, Walston JD, Segev DL. Frailty and falls among adult patients undergoing chronic hemodialysis: a prospective cohort study. $\mathrm{BMC} \mathrm{Ne}$ phrology 2013; 14:224.

18. Roshanravan B, Kathri M, Robinson-Cohen C, Levin G, Patel KV, De Boer IH, Seliger S, Ruzinski J, Himmelfarb J, Kestenbaum B. A prospective study of frailty in Nephrology-Referred patients with CKD. Am J Kidney Dis 2012; 60(6): 912-921.

19. Bao Y, Dalrymple L, Chertow GM, Kaysen GA, Johansen KL. Frailty, dialysis initiation, and mortality in end-stage renal disease. Arch Intern Med 2012; 172(24):1071-1077.

20. Reese PP, Cappola AR, Shults J, Townsend RR, Gadegbeku CA, Anderson C, Baker JF, Carlow D, Sulik MJ Lo JC, Go AS, Ky B, Mariani L, Feldman HI, Leonard MB; CRIC Study Investigators. Physical performance and frailty in chronic kidney disease. Am J Nephrol 2013; 38(4):307-315.

21. Mansur HN, Colugnati FAB, Grincenkov FRS, Bastos MG. Frailty and quality of life: a cross-sectional study of Brazilian patients with pre-dialysis chronic kidney disease. Health Qual Life Outcomes 2014; 12:27.

22. Johansen KL, Dalrympe LS, Delgado C, Kaysen GA, Kornak G, Grimes B, Chertow GM. Association between body composition and frailty among prevalent hemodialysis patients: A US Renal Data System Special Study. J Am Soc Nephrol 2014; 25(2):381-389.

Artigo apresentado em 23/08/2018

Aprovado em 07/03/2019

Versão final apresentada em 09/03/2019 
